\title{
Expression of E-cadherin and $\alpha$-catenin in a varicocele-induced infertility rat model
}

\author{
Hong Koo Ha ${ }^{1}$, Hyun Jun Park ${ }^{1,2}$ and Nam Cheol Park ${ }^{1,2}$
}

The roles of E-cadherin and $\alpha$-catenin were evaluated in the development of varicocele-induced infertility. Analysis of the association between the expression of E-cadherin/o-catenin and clinical/pathological parameters was performed. Thirty 10-week-old male rats (experimental group) were used for the experiments; the left renal vein was ligated to form a varicocele. The abdomen was incised in 30 rats (control group) and no procedure was performed on 10 rats (baseline group). The weights of the left testis, serum reactive oxygen species (ROS), testosterone, luteinizing hormone (LH), follicle-stimulating hormone (FSH) and degenerative changes in the seminiferous tubules after 4 and 8 weeks were recorded. The expression of E-cadherin and $\alpha$-catenin was evaluated by immunohistochemical (IHC) staining and Western blot analysis. The ROS increased in the 8-week experimental group, compared with the baseline and control groups ( $\boldsymbol{P}<0.001$ for both). Additionally, FSH significantly increased in the 4- and 8-week experimental group compared with the control groups $(P=0.013$ and $P=0.032$, respectively). The ratio of degenerative changes in the seminiferous tubules of the experimental groups increased. The IHC staining showed that the expression of E-cadherin and $\alpha$-catenin decreased in the 4- and 8-week experimental groups. Similar to the IHC staining, the experimental group had decreased reactivity on Western blot analysis. The expression of E-cadherin and $\alpha$-catenin was significantly associated with the ROS and degenerative changes in the seminiferous tubules. The results of this study suggest that damage to the blood-testis barrier (BTB) is associated with varicocele-induced male infertility, and that ROS may cause damage to the BTB.

Asian Journal of Andrology (2011) 13,470-475; doi:10.1038/aja.2010.94; published online 14 March 2011

Keywords: $\alpha$-catenin; blood-testis barrier; E-cadherin; varicocele; infertility

\section{INTRODUCTION}

A varicocele is a dilated internal spermatic vein that drains from the testis; it is found in $15 \%$ of young adults. Varicoceles are the cause of infertility in $35 \%$ of patients with primary infertility and in $81 \%$ of patients with secondary infertility. ${ }^{1}$ The mechanisms of testicular damage in patients with varicoceles include alteration of the hypothalamus-pituitary-gonadal axis, cell apoptosis and reactive oxygen species (ROS). ${ }^{2-5}$

Some investigators have recently suggested that the mechanisms correlated with testicular damage are also, at least in part, associated with injury to the blood-testis barrier (BTB). ${ }^{6}$ The BTB is composed of myoid cells and tight junctions (TJs) between the Sertoli cells; it separates the germ cells from the lymphatics and blood vessels of the testis. Wu et al. ${ }^{7}$ reported the presence of E-cadherin at the BTB in the testis. Expression of E-cadherin was found to be increased before the development of adherens junctions (AJs), desmosomes and gap junctions; it was suggested that E-cadherin was important in the development of the BTB.

The $\alpha$-, $\beta$ - and $\gamma$-catenins link E-cadherin to the actin cytoskeleton, and are important for the maintenance of its role in cell-to-cell adhesion. Hirano et al. ${ }^{8}$ reported that cells lacking $\alpha$-catenin are unable to form stable AJs despite normal E-cadherin and $\beta$-catenin expression; however, AJs were stable when $\alpha$-catenin expression was restored by cDNA transfection. Thus, the role of E-cadherin and $\alpha$-catenin appears to be important for the maintenance of the BTB in the testis.

The aim of this study was to investigate the role of E-cadherin and $\alpha$ catenin in the development of varicocele-induced infertility. We analyzed the association between BTB damage and clinical factors, including testicular weight, degenerative changes in the seminiferous tubules, ROS and hormonal alterations.

\section{MATERIALS AND METHODS}

Experimental surgery

A midline incision was carried out under general anesthesia using pentobarbital (3 mg per $100 \mathrm{~g}$ ) on thirty 10-week-old SpragueDawley male rats (Samtako Bio Korea Inc., O-San, Korea). The left renal vein was exposed medial to the insertion of the spermatic vein and then ligated with a $0.8-\mathrm{mm}$ stainless steel probe to decrease the diameter of the left renal vein, forming a varicocele. The rats were killed after 4 or 8 weeks. Dilatation of the internal spermatic vein was identified during the killing procedure. Using the same method described for the experimental group, general anesthesia and a midline incision were carried out on 30 rats that were of the same age as those 
in the control group; however, in the control group, the wound was closed without performing a procedure. All animals were killed 4 or 8 weeks later. The baseline group, which included 10 rats that did not undergo any procedures, was also studied.

The experimental protocol was approved by the Animal Care and Use Committee of our institution and was in accordance with the Declaration of Helsinki and the guideline issued by the International Association for the Study of Pain.

\section{Testicular weight}

The weights of the left testis from the baseline, 4-week control and experimental groups, and from the 8-week control and experimental groups were measured using a Mettler PM 460 (DeltaRange; Mettler Instrument Corp., Highstown, NJ, USA).

\section{Serum ROS}

The serum ROS was studied to assess varicocele-induced oxidative stress in the testis. Extraction of $20 \mu \mathrm{l}$ of serum was performed from the blood sample obtained from the internal spermatic vein, and the serum sample was added to microtubes with chromogen buffer (an amine derivative, reagents $\mathrm{R} 1$ of the FORT kit; $\mathrm{CrNH}_{2}$ ) and centrifuged at $1646.4 \mathrm{~g}$ for $1 \mathrm{~min}$ with $0.26 \mathrm{mg} \mathrm{l}^{-1} \mathrm{H}_{2} \mathrm{O}_{2}$ FORT units. The optical density was determined using a microplate reader at $505 \mathrm{~nm}$ (Form CR 2000; Callegari, Parma, Italy).

\section{Hormone assays}

The serum testosterone, luteinizing hormone ( $\mathrm{LH})$ and follicle-stimulating hormone (FSH) were measured before surgery and after the killing procedure in the baseline, experimental and control group samples obtained from the left renal vein by radioimmunoassay (WALLAC 1470 Wizard Automatic Gamma Counter; WALLAC Inc., Gaithersburg, MD, USA).

\section{Testicular pathology}

The testicular tissue was fixed with Bouin's fixative solution for 12 $24 \mathrm{~h}$, and the tissues were then set in paraffin wax. Hematoxylin and eosin staining was performed, and the ratio of the degenerative tubules to the normal tubules was evaluated under 200-fold magnification. Degenerative changes were defined as cell destruction or intercellular abnormalities. The slides were examined by two independent, experienced pathologists. Less than $10 \%, 10 \%-90 \%$ and greater than $90 \%$ were defined as normal, mild and severe degenerative changes, respectively.

Immunohistochemical (IHC) staining of E-cadherin and $\alpha$-catenin Subsequent to deparaffinization and rehydration, 4- $\mu \mathrm{m}$ testicular sections were treated with $0.3 \% \mathrm{H}_{2} \mathrm{O}_{2}$ in methanol for 10 min to block endogenous peroxidase activity; rat serum was then added for $5 \mathrm{~min}$ as a protein block. The sections were incubated for $1 \mathrm{~h}$ at room temperature with E-cadherin and $\alpha$-catenin antiserum. After incubation, the antibody was visualized with avidin-biotin-horseradish peroxidase complex using a Universal LSAB Kit (DAKO Corp., Carprinteria, CA, USA). After counterstaining with hematoxylin, the sections were dehydrated in graded ethanol rinses, cleared in xylene and coverslipped with Permount. For negative controls, primary antibody was replaced with non-immune IgG. Slides with histologically normal testis were used as positive controls.

The results of the IHC staining were classified according to the proportion of positive staining at the junctions between adjacent Sertoli cells. Staining was regarded as evidencing strong expression when a minimum of $91 \%$ of the junctions between adjacent Sertoli cells showed clear, membrane-associated staining. Staining was regarded as evidencing moderate expression when $11 \%-90 \%$ of the junctions between adjacent Sertoli cells were positively stained; staining was considered to indicate weak expression when $1 \%-10 \%$ of the junctions between adjacent Sertoli cells were stained. ${ }^{9}$ Slides were examined by two independent, experienced pathologists with no previous information on the tissue specimens (under $\times 100$ and $\times 200$ magnifications).

\section{Immunoblot analysis}

The tissues were homogenized in protein extraction solution (PROPREP; iNtRON Biotechnology Inc., Gyeonggi-do, Korea) and placed on ice for $30 \mathrm{~min}$. The suspensions were collected after centrifugation $\left(19353.6 \mathrm{~g}, 10 \mathrm{~min}, 4^{\circ} \mathrm{C}\right)$. The protein concentrations were quantified using the BCA Protein Assay Kit (Thermo Fisher Scientific Inc., Waltham, MA, USA) according to the manufacturer's instructions. Extracted proteins were resolved in SDS-polyacrylamide gels (8\% gel) and transferred onto a nitrocellulose membrane (HybondECL; GE Healthcare, Little Chalfont, UK) by electroblotting. Nitrocellulose blots were blocked with $5 \%$ non-fat dry milk for $1 \mathrm{~h}$ in Tris-Buffered Saline Tween-20 buffer $\left(20 \mathrm{mmol}^{-1}\right.$ Tris ( $\left.\mathrm{pH} 7.4\right)$, $137 \mathrm{mmol} \mathrm{l}^{-1} \mathrm{NaCl}$ and $0.1 \%$ Tween-20) at room temperature. Blots were incubated overnight at $4{ }^{\circ} \mathrm{C}$ with primary antibodies $(\alpha$-catenin, E-cadherin and glyceraldehyde-3-phosphate dehydrogenase; BD Bioscience, San Jose, CA, USA) in Tris-Buffered Saline Tween-20 buffer. Immunoreactive bands were detected using anti-mouse peroxidase-conjugated secondary antibody (GE Healthcare) and visualized by enhanced chemiluminescence (ECL Detection Kit; GE Healthcare). Each expression was calculated by height, area and optical density of the bands using a gel imaging system (UVIPro; UVItec Limited, Cambridge, UK).

\section{Statistical analyses}

Numerical data are expressed as mean \pm s.e. Statistical differences between means for the different groups were evaluated using the SPSS software (version 15.0 for Windows; SPSS Inc., Chicago, IL, USA), with the repeated measures analysis of variance for comparisons among groups, and followed by a post hoc Tukey's test. The correlation between clinical/pathological factors and E-cadherin and $\alpha$-catenin was evaluated by the Pearson's coefficient, with the level of significance set at a $P$ value $<0.05$. The statistical analysis of each experimental group with baseline and control groups is described.

\section{RESULTS}

\section{Testicular weight}

There was no significant difference among the baseline, control and experimental groups (Table $\mathbf{1}$ ).

\section{Serum ROS}

The 8-week experimental group showed a significant increase in the ROS levels compared with the baseline and 8-week control group $(P<0.001$ for both) (Table 1$)$.

\section{Hormone assays}

The serum FSH significantly increased in the experimental group compared with the control group $(P<0.005)$. However, there was no significant difference in the serum testosterone or serum $\mathrm{LH}$ among the baseline, control and experimental groups (Table 1). 
Table 1 Serum ROS, T, LH, FSH and degenerative change in seminiferous tubule in control and experimental groups

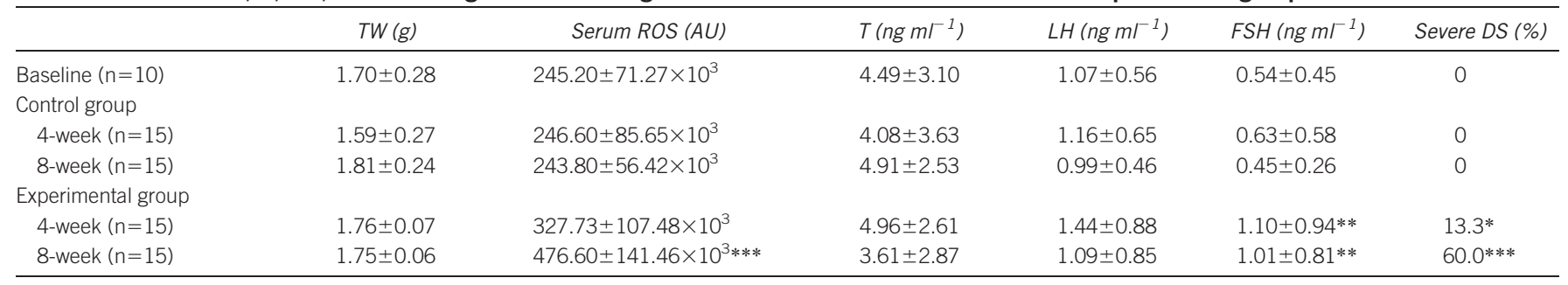

Abbreviations: AU, arbitrary units; DS, degenerative change in seminiferous tubule; FSH, follicle-stimulating hormone; LH, luteinizing hormone; ROS, reactive oxygen species; T, testosterone; TW, testicular weight.

$* P<0.005, * * * P<0.001$ compared with baseline and the corresponding control groups. ** $P<0.005$, compared with control groups.

\section{Degenerative change in the seminiferous tubules}

The ratio of degenerative change significantly increased in the experimental groups compared with the control groups (Table 1). Normal tubule grades were demonstrated in $90.0 \%, 93.3 \%, 86.7 \%, 60.0 \%$ and $6.7 \%$ in the baseline, 4 - and 8-week control and 4- and 8-week experimental groups, respectively. Mild degenerative grades were found in $10.0 \%, 6.7 \%, 13.3 \%, 26.7 \%$ and $33.3 \%$ and severe degenerative grades in $0,0,0,13.3 \%$ and $60.0 \%$, respectively. Degenerative changes in the 4-week $(P=0.021$ and $P=0.046)$ and 8 -week $(P<0.001$ for both) experimental groups increased significantly, compared with the baseline and control groups.

\section{Expression of E-cadherin and $\alpha$-catenin}

IHC staining of E-cadherin. The IHC staining for E-cadherin was significantly decreased in the 4 -week experimental group $(P=0.001$ and $P=0.001)$ and the 8 -week experimental group $(P<0.001$ and $P<0.001)$ compared with the baseline and control groups, respectively (Figures 1 and 2).

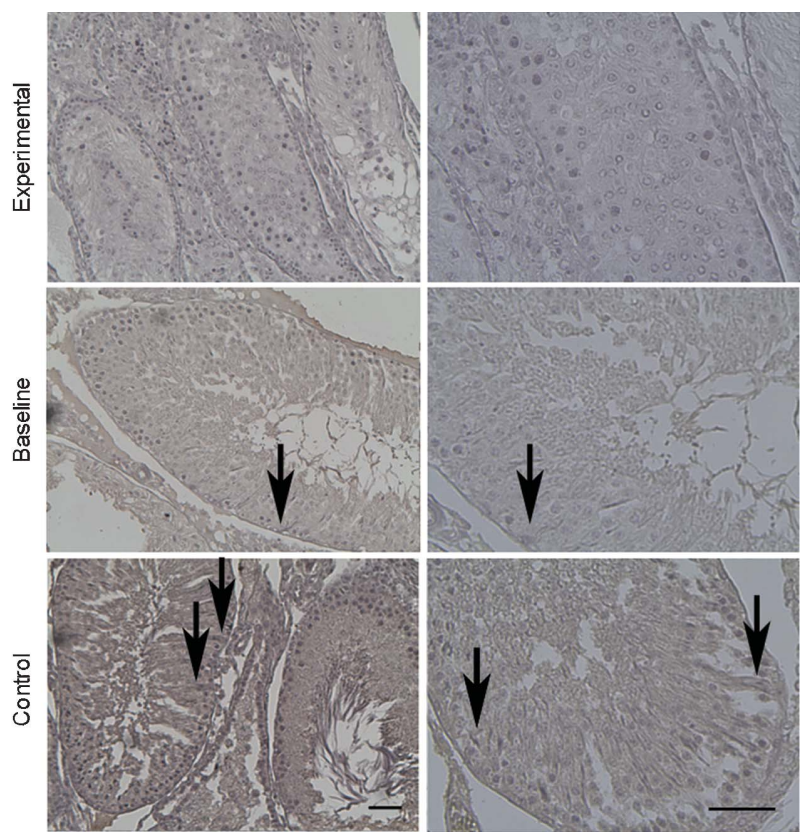

Figure 1 Immunohistochemical staining for E-cadherin at 8 weeks. E-cadherin immunoreactivity was shown (arrows). Weak stains were usually found in experimental groups and strong stains in the baseline and control groups. Scale bars $=50 \mu \mathrm{m}$.
IHC staining of $\alpha$-catenin. The IHC staining for $\alpha$-catenin was significantly decreased in the 4-week experimental group $(P=0.023$ and $P=0.001)$ and the 8 -week experimental group $(P<0.001$ and $P<0.001)$ compared with the baseline and control groups, respectively (Figures 3 and 4).

\section{Immunoblot analysis}

Western blot analysis of E-cadherin. The expression of E-cadherin on Western blot analysis by enhanced chemiluminescence and the results were: $100 \pm 31,100 \pm 28,100 \pm 34,58 \pm 17$ and $36 \pm 19$ in the baseline group, 4- and 8-week control, and 4- and 8-week experimental groups, respectively (Figure 5). The expression significantly decreased in the 4 - and 8-week experimental groups, compared with the baseline and control groups $(P<0.001$ and $P<0.001)$.

Western blot analysis of $\alpha$-catenin. The expression of $\alpha$-catenin on Western blot analysis by enhanced chemiluminescence (Western blot gel picture not shown) and the results were: $83 \pm 35,76 \pm 35,91 \pm 35$, $55 \pm 22$ and $27 \pm 14$ in the baseline group, 4 - and 8-week control, and 4and 8-week experimental groups, respectively. The expression significantly decreased in the 8-week experimental group, compared with the baseline and 8 -week control groups $(P=0.004$ and $P=0.028)$. The expression significantly decreased in the 4 - and 8 -week experimental group, compared with the baseline and control group $(P<0.001$ and $P<0.001)$.

Association between the expression of E-cadherin or $\alpha$-catenin and clinical factors

The expression of the IHC staining of E-cadherin showed significant correlation with the degenerative changes in the tubules $(P<0.001)$,

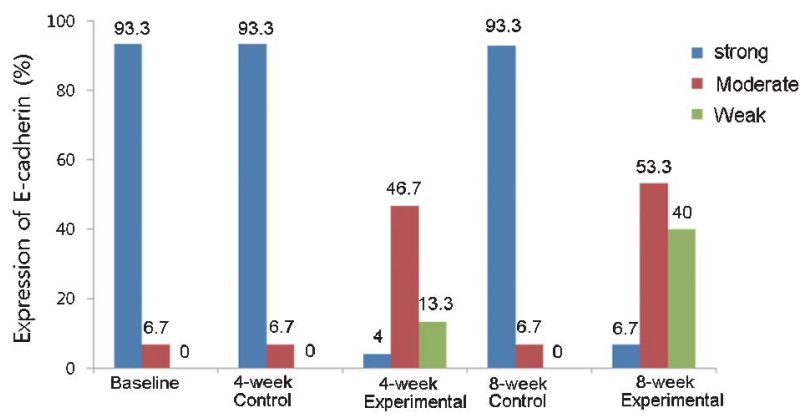

Figure 2 Expression of E-cadherin in each group. Strong staining was observed predominantly in the baseline, 4- and 8-week control groups. However, weak and moderate staining were significantly identified in the 4-week $(P=0.001$ and $P=0.001)$ and 8 -week $(P<0.001$ and $P<0.001)$ experimental groups compared with the baseline and control groups, respectively. 

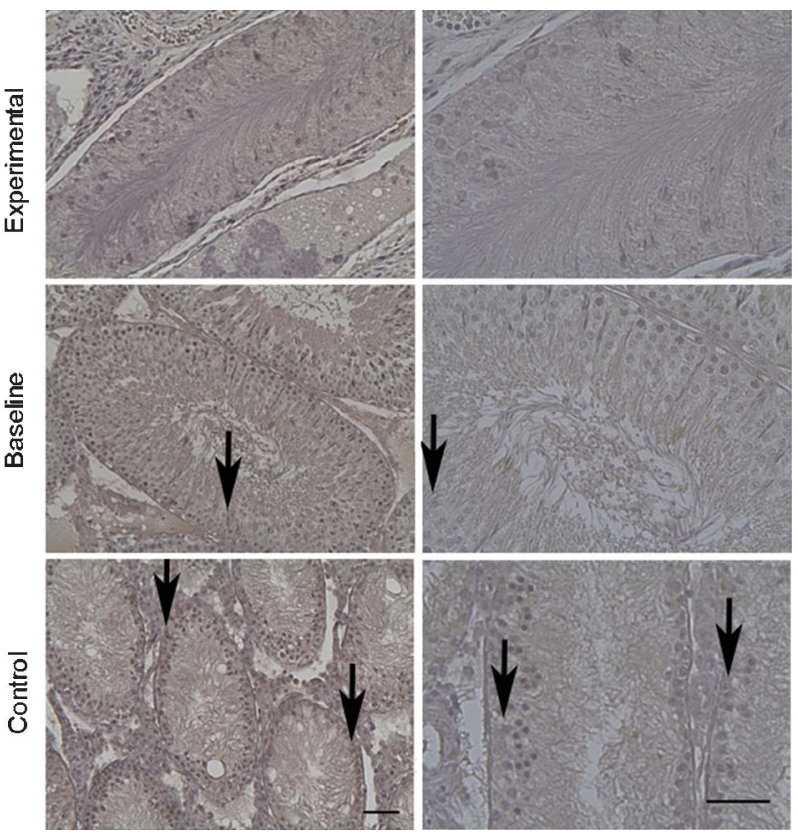

Figure 3 Immunohistochemical staining of $\alpha$-catenin at 8 weeks. $\alpha$-catenin immunoreactivity was shown (arrows). Similar to E-cadherin, weak stains were usually noted in the experimental groups and strong stains in the baseline and control groups. Scale bars $=50 \mu \mathrm{m}$.

ROS $(P<0.001)$ and FSH $(P=0.034)$. Similar to E-cadherin, the expression of the IHC staining of $\alpha$-catenin was significantly associated with the degenerative changes of the tubules $(P<0.001)$ and ROS $(P<0.001)$, but showed no correlation with other factors.

The expression of E-cadherin on Western blot analysis showed a significant relationship with the degenerative changes in the tubules $(P<0.001)$, ROS $(P<0.001)$ and FSH $(P=0.018)$. Similar to E-cadherin, the expression of $\alpha$-catenin on Western blot analysis was significantly associated with degenerative changes in the tubules $(P<0.001)$ and ROS $(P=0.003)$, but showed no correlation with other factors (Table 2).

\section{DISCUSSION}

The BTB, which is composed of TJs between Sertoli cells, is the densest barrier in testis tissue and shows highly dynamic cell structures. For example, preleptotene spermatocytes must translocate across the BTB, entering the adluminal compartment for further development, where they differentiate into pachytene spermatocytes. ${ }^{10}$ Thus, the BTB must disassemble to facilitate preleptotene spermatocyte movement; it is conceivable that extensive turnover of proteins occurs at the BTB during spermatogenesis.

Associations between Sertoli cells and germ cells facilitate germ cell translocation across the BTB via mechanisms of disassembly and

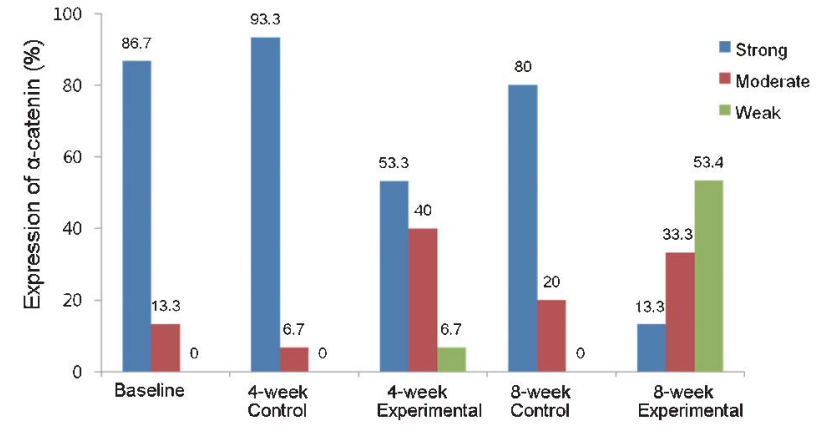

Figure 4 Expression of $\alpha$-catenin in each group. Strong staining was observed predominantly in the baseline, 4- and 8-week control groups. However, weak and moderate staining were significantly identified in 4-week ( $P=0.023$ and $P=0.001)$ and 8 -week $(P<0.001$ and $P<0.001)$ experimental groups compared with the baseline and control groups, respectively.

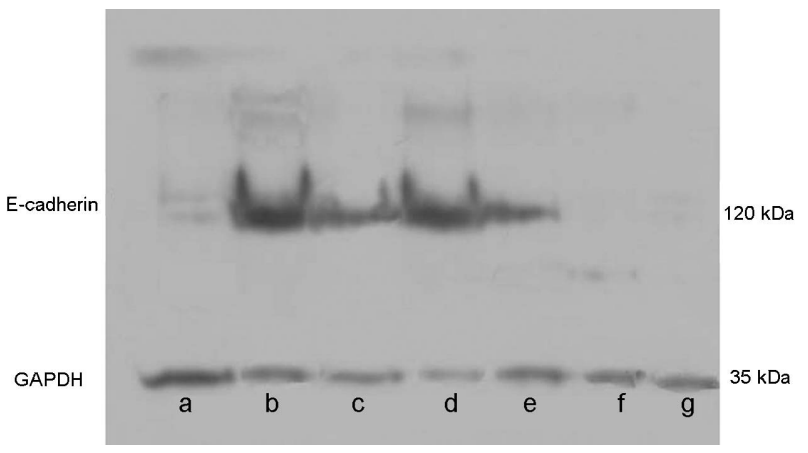

Figure 5 Expression of E-cadherin on Western blot analysis of each group. The expression of E-cadherin was observed as strong in the baseline and control groups; however, it was observed as moderate expression in the 4-week experimental group and weak expression in the 8-week experimental group (Lanes a, $f$ and g, 8-week experimental; lane b, 8-week control; lanes c and e, 4-week experimental; and lane d, baseline group). Samples were placed to the lane randomly to reduce experimenter's subjective effect.

assembly in the AJs and TJs. The AJ links cytoskeletal elements from either one cell to another cell or to the extracellular matrix, creating a network that maintains tissue integrity. Proleptotene/leptotene spermatocytes must cross this junction to become mature sperm; however, if the ability to cross the junction is disabled, infertility may occur. Because germ cells must migrate from the baseline to the adluminal compartments of the seminiferous epithelium, while remaining attached to the epithelium, the events of junctional dynamics and their regulation in the testes are among the most intriguing phenomena in spermatogenesis. ${ }^{11}$ How these events are regulated in the testes remains largely unknown. However, if the functionality of these junctions is disrupted or compromised, germ cells can no longer attach to

Table 2 Relationships between expression of E-cadherin/ $\alpha$-catenin and clinicopathological parameters by Pearson coefficients

\begin{tabular}{|c|c|c|c|c|c|c|}
\hline & $T W$ & ROS & $L H$ & FSH & $T$ & $D C$ \\
\hline \multicolumn{7}{|c|}{ Immunohistochemical staining } \\
\hline E-cadherin & $0.112(P=0.394)$ & $0.552(P<0.001)$ & $0.132(P=0.314)$ & $0.274(P=0.034)$ & $-0.045(P=0.736)$ & $0.514(P<0.001)$ \\
\hline$\alpha$-catenin & $0.041(P=0.755)$ & $0.519(P<0.001)$ & $0.010(P=0.940)$ & $0.226(P=0.082)$ & $-0.163(P=0.213)$ & $0.507(P<0.001)$ \\
\hline \multicolumn{7}{|c|}{ Western blotting } \\
\hline E-cadherin & $0.047(P=0.720)$ & $-0.488(P<0.001)$ & $-0.034(P=0.798)$ & $-0.304(P=0.018)$ & $0.065(P=0.622)$ & $-0.540(P<0.001)$ \\
\hline$\alpha$-catenin & $-0.035(P=0.788)$ & $-0.373(P=0.003)$ & $-0.155(P=0.235)$ & $-0.099(P=0.451)$ & $0.219(P=0.093)$ & $-0.495(P<0.001)$ \\
\hline
\end{tabular}

Abbreviations: DC, degenerative change; FSH, follicle-stimulating hormone; LH, luteinizing hormone; ROS, reactive oxygen species; T, testosterone; TW, testicular weight. 
the epithelium and will be removed from the testes, which could result in infertility. ${ }^{12,13}$

Cadherins are transmembrane proteins of $115-130 \mathrm{kDa} .{ }^{14}$ The cadherin family includes epithelial, placental and neural cadherin and liver cell adhesion molecules. ${ }^{15,16}$ E-cadherin is a $120-\mathrm{kDa}$ protein. Intracellular $\mathrm{COOH}$-terminal domains of cadherins associate with $\beta$ - or $\gamma$-catenin at the site of AJs, forming the cadherin/catenin complex, whereas the extracellular $\mathrm{NH}_{2}$-terminal domains of two cadherins residing in adjacent cells interact homotypically. These complexes are functional cell adhesive units. ${ }^{17}$ In turn, $\alpha$-catenin interacts with $\beta$ or $\gamma$-catenin-bound cadherin, linking this complex to the actin cytoskeleton. ${ }^{18}$ The interaction of cadherin and catenin requires calcium ions and is regulated by GTPase. ${ }^{19}$ Thus, in the absence of calcium ions, cadherins are inactive and susceptible to proteolysis.

Catenins were first identified as a set of proteins that could immunoprecipitate with E-cadherin ${ }^{20}$ and were similar in structure. ${ }^{21}$ The $\beta$-catenin binds directly to the cadherin cytoplasmic tail, ${ }^{22}$ whereas $\alpha$ catenin binds to $\beta$-catenin and connects the complex to actin or actinin. ${ }^{17}$ Cells lacking $\alpha$-catenin are unable to form stable contacts despite high expression levels of E-cadherin and $\beta$-catenin. ${ }^{8}$ Moreover, interaction of $\alpha$-catenin with the actin-based cytoskeleton through the ZO-1-binding domain is required for a strong state of E-cadherinbased cell adhesion activity. ${ }^{23}$ It is also known that binding of $\alpha$-catenin to ZO-1 can affect the strength of E-cadherin-mediated adhesion in non-epithelial cells, suggesting that the role of catenins in different cell types could be different. ${ }^{23}$ In the case of defects in $\alpha$-catenin, despite normal E-cadherin and $\beta$-catenin, the AJs are unstable. Moreover, interaction between $\alpha$-catenin and the cytoskeleton via ZO-1 is important for the adhesion activity of E-cadherin. ${ }^{23}$ These findings suggest that $\mathrm{E}$-cadherin and $\alpha$-catenin are important for the maintenance of BTB function.

Antisperm antibodies have been suggested as another mechanism in which BTB disruption may have a role in the pathophysiology of the varicocele-induced male infertility. Owing to an abnormality of the junctional structure between Sertoli cells and germ cells in patients with varicoceles, it has been suggested that the BTB was associated with the pathophysiology of a varicocele. ${ }^{24}$ However, there is not enough information on E-cadherin and $\alpha$-catenin expression at the junctions between adjacent Sertoli cells of testicular specimens from patients with varicoceles to determine whether there is any link between the BTB and the pathophysiology of varicoceles. Koksal et al. ${ }^{9}$ suggested that reduced E-cadherin and $\alpha$-catenin expression at the junctions between adjacent Sertoli cells may be associated with disruption of the BTB. In this study, the expression of E-cadherin and $\alpha$-catenin decreased in the experimental group compared with the control group.

Sperm analysis has been a useful method for evaluation of varicocele-induced testicular damage. However, a varicocele is usually diagnosed in adolescents; thus, most clinicians recommend use of testicular size as criteria for surgery due to psychological and/or ethical problems. In recent studies, however, the testicular weight of the affected testis in patients with varicoceles did not show a larger decrease than the testicular weight of the contralateral side, according to the grade of the varicocele. ${ }^{25}$ This study also showed that the testicular size in rats with a varicocele did not decrease more than that in the rats in the control group. However, increased degenerative changes in the rats with a varicocele suggest that the varicocele did cause testicular damage.

There have been large studies on ROS and infertility. ${ }^{3}$ ROS can attack all cells in the human body; sperm in particular are susceptible to ROS owing to their abundant, unsaturated lipid content. ${ }^{26}$ ROS usually induces apoptosis of sperm through direct DNA disruption or through alteration of the concentration of intercellular calcium ions. ${ }^{4,27-29}$ The alteration of intracellular ion concentration may affect the mechanisms associated with TJs or AJs. The findings in this study of increased ROS in the varicocele group support the association between varicoceles and ROS.

Abnormalities of hormones associated with a varicocele are important factors that correlate with the development of infertility. Camoglio et $a l^{30}$ reported decreased testosterone and increased FSH, but no change in the LH of patients with a varicocele. However, in this study, these hormones were not significantly altered, except FSH. These findings may be owing to the short experimental period.

In the current study, there were significant increases in the degenerative changes in the seminiferous tubules between the 4-week experimental and control groups; however, no significant difference in ROS was observed between the groups. The ROS was significantly different between the 8-week experimental and control groups. These results suggest that the mechanism of testicular damage may differ according to the duration of the varicocele.

Expression of E-cadherin and $\alpha$-catenin was significantly associated with degenerative changes in the seminiferous tubules. Degenerative changes associated with infertility, and E-cadherin and $\alpha$-catenin suggest that they are important proteins in the BTB. Because spermatocytes must cross the BTB to mature, abnormalities in the TJ or AJ can cause infertility. Thus, expression of E-cadherin and $\alpha$-catenin are good indices of infertility. As mentioned previously, there have been a few reports on the association between cadherin/catenin and infertility; some investigators have suggested anti-cadherin/anti-catenin antibodies for male contraception. ${ }^{31}$ However, additional studies are required to define the association between the cadherin/catenin complex and infertility associated with a varicocele. ROS was significantly associated with the expression of E-cadherin and $\alpha$-catenin in this study. Because the ROS disrupts the cell membrane, the transmembrane proteins, cadherin and catenin, may also be susceptible to ROS. ROS can damage Sertoli cells and may induce disruption of the BTB through alteration of expression of cadherin and catenin. Additionally, ROS can disrupt the AJs of spermatocytes, causing difficulty in adhesion between Sertoli cells and preleptotene/leptotene spermatocytes, leading to infertility.

Infertile men have a higher frequency of antisperm antibodies than do fertile men. Infertile men with a varicocele have a level of antisperm antibodies that is similar to that of infertile men without a varicocele. ${ }^{32}$ In rats with experimentally induced varicoceles, a higher antisperm antibody level was reported than in sham and non-operated rats. ${ }^{33}$ However, results from earlier studies demonstrated that the status of the BTB in varicocele pathophysiology remains unclear. Raitsina et al. ${ }^{34}$ showed that disturbance of permeability and of the fine BTB structure in both testes were observed in testes affected with a varicocele, and that the morphological change was similar to that in autoimmune orchitis.

In the current study, changes in the expression of E-cadherin and $\alpha$-catenin were found in the varicocele. This suggests that altered expression of E-cadherin and $\alpha$-catenin may cause abnormal transportation of spermatocytes and, eventually, infertility. However, the mechanisms that cause the changes associated with a varicocele and the protein interactions in the BTB have not been determined. The clinical application for contraception is theoretically possible but requires further study.

E-cadherin and $\alpha$-catenin are important for the maintenance of the BTB, and the BTB has an important role in spermatogenesis. Thus, 
decreased expression of these proteins may be associated with infertility. In this study, the expression of E-cadherin and $\alpha$-catenin was decreased, and this expression correlated with ROS and degenerative changes in the seminiferous tubules in a varicocele-induced rat model. These results suggest that the varicocele induces ROS, and the increased ROS causes damage to the BTB by alteration of the intracellular ionic concentration or direct damage to sperm. Thus, expression of E-cadherin and $\alpha$-catenin may be a useful index for assessment of testicular damage in the varicocele-induced rat model. The role of ROS in transmembrane proteins, particularly the cadherin/catenin complex, has not been determined. The mechanisms by which cadherins and catenins may be associated with ROS and infertility require further evaluation.

\section{AUTHOR CONTRIBUTIONS}

HJP designed the study. HJP and HKH carried out the experiment. HKH collected and analyzed data. HKH and NCP interpreted the data and wrote the paper.

\section{COMPETING FINANCIAL INTERESTS}

The authors declare no competing financial interests.

\section{ACKNOWLEDGMENTS}

This study was supported by a Medical Research Institute Grant (No. 2008-16) from Pusan National University Hospital, Korea.

1 Gorelick JI, Goldstein M. Loss of fertility in men with varicocele. Fertil Steril 1993; 59 613-6.

2 Fujisawa M, Hiramine C, Tanaka H, Okada H, Arakawa S et al. Decrease in apoptosis of germ cells in the testes of infertile men with varicocele. World J Urol 1999; 17: 296300 .

3 Hendin BN, Kolettis PN, Sharma RK, Thomas AJ Jr, Agarwal A. Varicocele is associated with elevated spermatozoal reactive oxygen species production and diminished seminal plasma antioxidant capacity. J Urol 1999; 161: 1831-4.

$4 \mathrm{Ku} \mathrm{JH}$, Shim HB, Kim SW, Paick JS. The role of apoptosis in the pathogenesis of varicocele. BJU Int 2005; 96: 1092-6.

5 Naughton CK, Nangia AK, Agarwal A. Pathophysiology of varicoceles in male infertility. Hum Reprod Update 2001; 7: 473-81.

6 Mruk DD, Cheng CY. Sertoli-Sertoli and Sertoli-germ cell interactions and their significance in germ cell movement in the seminiferous epithelium during spermatogenesis. Endocr Rev 2004; 25: 747-806.

7 Wu JC, Gregory CW, DePhilip RM. Expression of E-cadherin in immature rat and mouse testis and in rat Sertoli cell cultures. Biol Reprod 1993; 49: 1353-61.

8 Hirano S, Kimoto N, Shimoyama Y, Hirohashi S, Takeichi M. Identification of a neura alpha-catenin as a key regulator of cadherin function and multicellular organization. Cell 1992; 70: 293-301.

9 Koksal IT, Ishak Y, Usta M, Danisman A, Guntekin E et al. Varicocele-induced testicular dysfunction may be associated with disruption of blood-testis barrier. Arch Androl 2007; 53: 43-8.

10 Lie PP, Xia W, Wang CQ, Mruk DD, Yan HH et al. Dynamin II interacts with the cadherin- and occludin-based protein complexes at the blood-testis barrier in adult rat testes. J Endocrinol 2006; 191: 571-86.
11 Byers SW, Sujarit S, Jegou B, Butz S, Hoschutzky H et al. Cadherins and cadherinassociated molecules in the developing and maturing rat testis. Endocrinology 1994 134: 630-9.

12 Chung NP, Mruk D, Mo MY, Lee WM, Cheng CY. A 22-amino acid synthetic peptide corresponding to the second extracellular loop of rat occludin perturbs the bloodtestis barrier and disrupts spermatogenesis reversibly in vivo. Biol Reprod 2001 65: 1340-51.

13 Grima J, Silvestrini B, Cheng CY. Reversible inhibition of spermatogenesis in rats using a new male contraceptive, 1-(2,4-dichlorobenzyl)-indazole-3-carbohydrazide. Biol Reprod 2001; 64: 1500-8

14 Takeichi M. Cadherins: a molecular family important in selective cell-cell adhesion. Annu Rev Biochem 1990; 59: 237-52.

15 Christofori G, Semb H. The role of the cell-adhesion molecule E-cadherin as a tumoursuppressor gene. Trends Biochem Sci 1999; 24: 73-6.

16 Guilford P. E-cadherin downregulation in cancer: fuel on the fire? Mol Med Today 1999; 5: 172-7

17 Gumbiner BM. Regulation of cadherin adhesive activity. J Cell Biol 2000; 148: 399 404.

18 Rimm DL, Koslov ER, Kebriaei P, Cianci CD, Morrow JS. Alpha 1(E)-catenin is an actin-binding and -bundling protein mediating the attachment of F-actin to the membrane adhesion complex. Proc Natl Acad Sci USA 1995; 92: 8813-7.

19 Huber O, Bierkamp C, Kemler R. Cadherins and catenins in development. Curr Opin Cell Biol 1996; 8: 685-91.

20 Gumbiner BM, McCrea PD. Catenins as mediators of the cytoplasmic functions of cadherins. J Cell Sci Supp/ 1993; 17: 155-8.

21 Kowalczyk AP, Bornslaeger EA, Norvell SM, Palka HL, Green KJ. Desmosomes intercellular adhesive junctions specialized for attachment of intermediate filaments. Int Rev Cytol 1999; 185: 237-302.

22 Huber AH, Weis WI. The structure of the beta-catenin/E-cadherin complex and the molecular basis of diverse ligand recognition by beta-catenin. Cell 2001; 105: 391 402.

23 Imamura Y, Itoh M, Maeno Y, Tsukita S, Nagafuchi A. Functional domains of alphacatenin required for the strong state of cadherin-based cell adhesion. J Cell Biol 1999; 144: 1311-22.

24 Cameron DF, Snydle FE, Ross MH, Drylie DM. Ultrastructural alterations in the adluminal testicular compartment in men with varicocele. Fertil Steril 1980; 33 526-33.

$25 \mathrm{Ku} \mathrm{JH}$, Son $\mathrm{H}$, Kwak C, Lee SE, Lee NK et al. Impact of varicocele on testicular volume in young men: significance of compensatory hypertrophy of contralateral testis. J Urol 2002; 168: 1541-4

26 Awda BJ, Mackenzie-Bell M, Buhr MM. Reactive oxygen species and boar sperm function. Biol Reprod 2009; 81: 553-61.

27 Agarwal A, Allamaneni SS. Sperm DNA damage assessment: a test whose time has come. Fertil Steril 2005; 84: 850-3.

28 Agarwal A, Said TM. Oxidative stress, DNA damage and apoptosis in male infertility: a clinical approach. BJU Int 2005; 95: 503-7.

29 Saleh RA, Agarwal A, Sharma RK, Said TM, Sikka SC et al. Evaluation of nuclear DNA damage in spermatozoa from infertile men with varicocele. Fertil Steril 2003; 80 1431-6.

30 Camoglio FS, Zampieri N, Corroppolo M, Chironi C, Dipaola G et al. Varicocele and retrograde adrenal metabolites flow. An experimental study on rats. Urol Int 2004; 73 337-42.

31 Hernandez Gifford JA, Hunzicker-Dunn ME, Nilson JH. Conditional deletion of betacatenin mediated by Amhr2cre in mice causes female infertility. Biol Reprod 2009; 80: 1282-92.

32 Golomb J, Vardinon N, Homonnai ZT, Braf Z, Yust I. Demonstration of antispermatozoal antibodies in varicocele-related infertility with an enzyme-linked immunosorbent assay (ELISA). Fertil Steril 1986; 45: 397-402.

33 Shook TE, Nyberg LM, Collins BS, Mathur S. Pathological and immunological effects of surgically induced varicocele in juvenile and adult rats. Am J Reprod Immunol Microbiol 1988; 17: 141-4.

34 Raitsina SS, Kurnosov AV, larovaia IM, Gladkova NS, Davydova Al. Autoimmune nature of spermatogenesis disorders in varicocele models in rats. Biull Eksp Biol Med 1979; 87: 44-8. 